\section{A Drosophila NSF mutant}

SIR - Recent work suggests that the molecular machinery mediating vesicle targeting and fusion is conserved in both constitutive and regulated secretion (reviewed in refs 1, 2). Here we report that the Drosophila mutant comatose (comt) is defective in one component of this conserved secretory apparatus, the $N$-ethylmaleimide-sensitive fusion protein (NSF). Furthermore, comt exhibits an apparent defect in synaptic transmission which is the first functional evidence that NSF is involved in this process.

NSF was initially identified as a protein required for vesicular transport in the constitutive secretory pathway present in all eukaryotic cells. The requirement for NSF in this process was shown to occur after vesicle formation and targeting, and thus NSF was proposed to participate in vesicle fusion, or a step closely preceding it. Subsequently, NSF was used to identify other proteins that function in vesicular transport, including the soluble NSF attachment proteins (SNAPs) ${ }^{3}$ and the SNAP receptors (SNAREs) ${ }^{4}$. Because SNAREs were found to be identical to previously characterized synaptic proteins required for synaptic transmission, NSF was implicated in this process as well.

We recently initiated a genetic analysis of synaptic transmission in the fruit fly Drosophila melanogaster by identifying neurally expressed Drosophila homologues of NSF and the SNAPs ${ }^{5}$. The gene encoding the NSF homologue $(d N S F)$ was localized to the $11 \mathrm{D} 9-\mathrm{E} 4$ region of the $\mathrm{X}$ chromosome. Candidate $d N S F$ mutations, uncovered by the same set of deletions that remove the $d N S F$ gene, were then identified among existing recessive mutations. Mutations of the comt locus, identified by Siddiqi and Benzer on the basis of their temperature-sensitive paralytic phenotype ${ }^{6}$, were among the candidate mutations mapped to this region $(\mathrm{K}$. S. Krishnan, personal communication).

To examine the possibility that comt

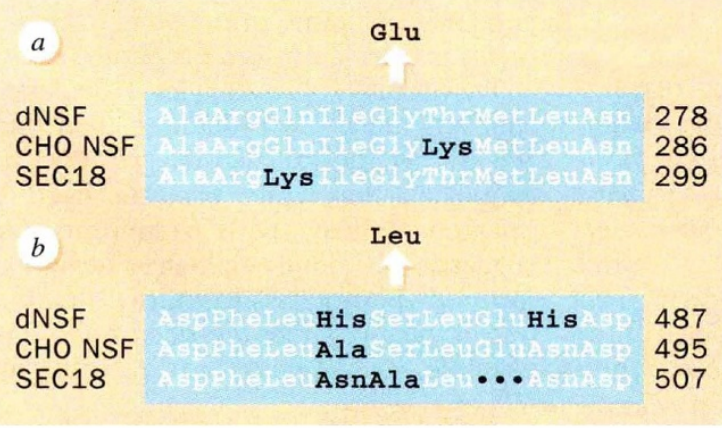

FIG. 1 dNSF missense mutations detected by sequence analysis of PCR-derived dNSF CDNAs from comt ${ }^{\mathrm{st17}}$ (a) and $\operatorname{comt}^{\mathrm{t} 53}(b)$ are indicated by the arrows. The relevant portion of the dNSF amino-acid sequence is shown in alignment with Chinese hamster ovary NSF and Saccharomyces cerevisiae SEC18. Amino-acid identities are highlighted.

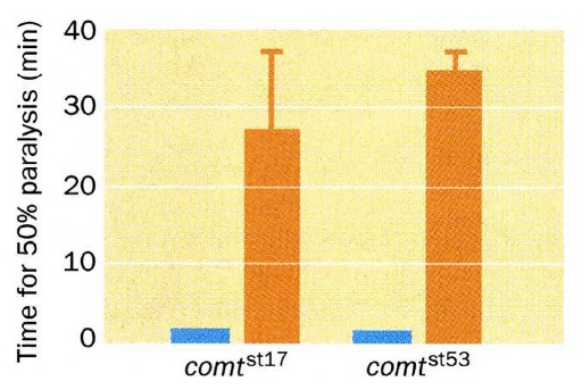

FIG. 2 Analysis of NSF transformants in the rescue of the comt temperature-sensitive paralytic phenotype. Flies heterozygous for a $\mathrm{P}$ element construct bearing a dNSF cDNA under the control of a heat-shock 70 promoter $^{7}$ were subjected to $38^{\circ} \mathrm{C}$ heat shocks of 15 and 30 min duration on successive days to induce expression of dNSF protein. Flies were tested for paralysis at $38{ }^{\circ} \mathrm{C} 24-28 \mathrm{~h}$ after the second heat-shock treatment (as described in ref. 6), but with the following modification: filter paper inserts in the bottom of the tubes used in the water bath test allowed slightly impaired flies to right themselves. As a control, nontransformant comt siblings from the same crosses that generated the test flies were subjected to the same heat-shock regime and examined for temperature-sensitive paralysis in identical fashion.

mutations are alterations in $d N S F$, PCR (polymerase chain reaction)-derived complementary DNAs containing the entire $d N S F$ open reading frame were obtained from two different comt alleles and wild-type flies, and sequenced. Both the comt $t^{\text {st17 }}$ and comt $t^{\mathrm{st53}}$ alleles contain a single missense mutation relative to the wild-type cDNA sequence (Fig. 1). Furthermore, both comt mutations are $\mathrm{G} \cdot \mathrm{C}$ to $\mathrm{A} \cdot \mathrm{T}$ transitions, the most common type of mutational change caused by ethylmethane sulphonate, the mutagen used to generate comt alleles.

As a more definitive test, transgenic flies were constructed to determine whether expression of wild-type dNSF protein can rescue the comt phenotype. A P-element transposon bearing a fulllength $d N S F$ cDNA, under the control of a heat-shock promoter, was used to confer heat-shock-inducible expression of dNSF protein. Rescue was assessed by examining the temperaturesensitive paralysis of comt flies, which normally occurs within 1-2 minutes of exposure to $38{ }^{\circ} \mathrm{C}$. After heat shock, followed by a 1-day recovery period, comt flies bearing the $d N S F$ transgene were highly resistant to exposure to $38^{\circ} \mathrm{C}$, while their comt siblings lacking the transgene remained sensitive (Fig. 2). Taken together with the mapping and sequence data, these results show that the comt gene is $d N S F$.

Our finding provides new information on the physiological role of NSF. Electrophysiological analysis of neuromuscular transmission in the flight muscles of comt mutants ${ }^{6}$ showed that nonpermissive temperatures cause a graded decrease in the postsynaptic potential, consistent with a defect in synaptic transmission. Thus, these findings represent the first functional evidence for NSF involvement in synaptic transmission. Further electrophysiological analysis of the comt mutant should clarify the role of NSF in this process.

\section{Leo Pallanck}

\section{Richard W. Ordway}

\section{Barry Ganetzky}

Laboratory of Genetics,

University of Wisconsin-Madison,

Madison, Wisconsin 53706, USA

1. Rothman, J. E. Nature 372, 55-63 (1994).

2. Ferro-Novick, S. \& Jahn, R. Nature 370, 191-193 (1994).

3. Whiteheart, S. W. et al, Nature 362, 353-355 (1993).

4. Söllner, T, et al. Nature 362, 318-324 (1993).

5. Ordway, R., Pallanck, L. \& Ganetzky, B. Proc. natn. Acad. Sci. U.S.A. 91, 5715-5719 (1994).

6. Siddiqi, O. \& Benzer, S. Proc. natn. Acad. Sci. U.S.A. 73, 3253-3257 (1976).

7. Karch, F., Török, I. \& Tissières, A. J. molec. Biol. 148, 219-230 (1981)

\section{Origins of photosynthesis}

SIR - Nisbet et al. ${ }^{1}$ in Scientific Correspondence have suggested that photosynthesis originated at hydrothermal vents, where organisms could exploit thermal radiation from the hot water. It is a mystery how primitive organisms could exploit sunlight before the appearance of ozone which provides protection from ultraviolet radiation. Here we attempt to expose some quantitative aspects not mentioned by Nisbet et al.

In their computations, Nisbet et al. consider water to be a black body. In reality, water itself should radiate more strongly in the absorption bands than at other wavelengths, and these bands are also those which would be most strongly attenuated by the cooler water between the radiation source and the organisms. The radiator does not, however, consist of pure water, and a 'black smoker' would be black enough, so the calculations below are also based on black-body radiation.

To investigate whether thermal radiation from vents would be able to drive biochemical and biophysical processes, I have computed the chemical potential difference that can be achieved using such radiation. As in a previous paper $^{2}$ in which I derived the optimum wavelength for the long-wavelength absorption band of a photosynthetic system in unattenuated daylight, I follow the approach of Ross and Calvin ${ }^{3}$. Lands- 